\title{
ON PROPERTIES OF SOME MATRIX SPLITTINGS*
}

\author{
HENRYK A. JEDRZEJEC ${ }^{\dagger}$ AND ZBIGNIEW I. WOŹNICKI ${ }^{\dagger}$
}

\begin{abstract}
Properties of some splittings of a monotone matrix $A$ are discussed. These properties are fundamental hypotheses in the proofs of comparison theorems. This fact is illustrated by several results given in the literature.
\end{abstract}

Key words. Systems of linear equations, convergence conditions, comparison theorems, nonnegative splittings, weak nonnegative splittings, weak splittings, weaker splittings.

AMS subject classifications. $65 \mathrm{C} 20,65 \mathrm{~F} 10,65 \mathrm{~F} 15$

This paper is closely related to [10]. The following definitions of splittings are used.

Definition 1.1. Let $M, N \in \mathbb{R}^{n \times n}$. Then the decomposition $A=M-N$ is called

(a) a regular splitting of $A$ if $M^{-1} \geq 0$ and $N \geq 0$,

(b) a nonnegative splitting of $A$ if $M^{-1} \geq 0, M^{-1} N \geq 0$ and $N M^{-1} \geq 0$,

(c) a weak nonnegative splitting of $A$ if $M^{-1} \geq 0$ and either $M^{-1} N \geq 0$ (the first type) or $N M^{-1} \geq 0$ (the second type),

(d) a weak splitting of $A$ if $M$ is nonsingular, $M^{-1} N \geq 0$ and $N M^{-1} \geq 0$,

(e) a weaker splitting of $A$ if $M$ is nonsingular and either $M^{-1} N \geq 0$ (the first type) or $N M^{-1} \geq 0$ (the second type),

(f) a convergent splitting of $A$ if $\varrho\left(M^{-1} N\right)=\varrho\left(N M^{-1}\right)<1$.

The splittings defined in the successive items extend successively a class of splittings of $A=M-N$ for which the matrices $N$ and $M^{-1}$ may lose the properties of nonnegativity. Distinguishing both types of weak nonnegative and weaker splittings leads to further extensions allowing us to analyze cases when $M^{-1} N$ may have negative entries even if $N M^{-1}$ is a nonnegative matrix.

It is necessary to mention that the definition assumed in item (b) is equivalent to the definition of weak regular splitting of $A$ introduced originally by Ortega and Rheinboldt [6]. However, some authors, for instance Berman and Plemmons [1], using the same name "weak regular splitting", restrict this definition to its weaker version based on the conditions $M^{-1} \geq 0$ and $M^{-1} N \geq 0$ without the condition $N M^{-1} \geq 0$, which corresponds to a weak nonnegative splitting of the first type defined in item (c).

In the literature there are many comparison theorems proven under different hypotheses for the splittings of the above definition.

Conditions ensuring that a splitting of $A=M-N$ is convergent are unknown in a general case. However, as is well known [8,9], the splittings defined in first three

\footnotetext{
${ }^{*}$ Received by the editors on 25 October 1999. Accepted for publication on 1 February 2001. Handling Editor: Miroslav Fiedler. This paper was presented at the Eleventh Haifa Matrix Theory Conference, Technion, Haifa, Israel, June 21-25, 1999.

$\dagger$ Institute of Atomic Energy 05-400 Otwock-Świerk, Poland (henj@hp2.cyf.gov.pl, woznicki@hp2.cyf.gov.pl).
} 
items of Definition 1.1 are convergent if and only if $A^{-1} \geq 0$.

The properties of weak nonnegative splittings are summarized in the following theorem.

TheOREm 1.2. [8] Let $A=M-N$ be a weak nonnegative splitting of $A$. If $A^{-1} \geq 0$, then:

1. $A^{-1} \geq M^{-1}$.

2. $\varrho\left(M^{-1} N\right)=\varrho\left(N M^{-1}\right)<1$.

3. If $M^{-1} N \geq 0$, then $A^{-1} N \geq M^{-1} N$ and if $N M^{-1} \geq 0$, then $N A^{-1} \geq N M^{-1}$.

4. $\varrho\left(M^{-1} N\right)=\frac{\varrho\left(A^{-1} N\right)}{1+\varrho\left(A^{-1} N\right)}=\frac{\varrho\left(N A^{-1}\right)}{1+\varrho\left(N A^{-1}\right)}<1$.

Conversely, if $\varrho\left(M^{-1} N\right)<1$, then $A^{-1} \geq 0$.

The relation in item 4 was obtained by Varga [7] for regular splittings of $A$. From the above theorem we can conclude the following corollary.

COROLlary 1.3. Each weak nonnegative (as well as nonnegative and regular) splitting of $A=M-N$ is convergent if and only if $A^{-1} \geq 0$. In other words, if $A$ is not a monotone matrix, it is impossible to construct a convergent weak nonnegative splitting.

It is obvious that when two weak nonnegative splittings of a monotone matrix $A=M_{1}-N_{1}=M_{2}-N_{2}$ (i.e., $A^{-1} \geq 0$ ) are of the same type, the inequality

$$
N_{2} \geq N_{1}
$$

implies either

$$
A^{-1} N_{2} \geq A^{-1} N_{1} \geq 0
$$

or

$$
N_{2} A^{-1} \geq N_{1} A^{-1} \geq 0 .
$$

Hence, we have $\varrho\left(A^{-1} N_{2}\right) \geq \varrho\left(A^{-1} N_{1}\right)$ and by Theorem 1.2 we can conclude that $\varrho\left(M_{1}^{-1} N_{1}\right) \leq \varrho\left(M_{2}^{-1} N_{2}\right)$.

Usually, for weak nonnegative splittings the matrices $M_{1}^{-1} N_{1} \geq 0$ or $N_{2} M_{2}^{-1} \geq 0$ are accompanied by the conditions $N_{1} A^{-1} \nsucceq 0$ or $A^{-1} N_{2} \nsucceq 0$, respectively. When both weak nonnegative splittings are of different types, one of the matrices $A^{-1} N_{2}$ and $A^{-1} N_{1}$ or $N_{2} A^{-1}$ and $N_{1} A^{-1}$ may have negative entries, which does not allow us to conclude that the inequality $\varrho\left(M_{1}^{-1} N_{1}\right) \leq \varrho\left(M_{2}^{-1} N_{2}\right)$ is satisfied. Let us assume that $M_{1}^{-1} N_{1} \geq 0$ and $N_{2} M_{2}^{-1} \geq 0$ which implies that $A^{-1} N_{1} \geq 0$ and $N_{2} A^{-1} \geq 0$, then from (1.1) we have

$$
A^{-1} N_{2} \geq A^{-1} N_{1} \geq 0 \quad \text { and } \quad N_{2} A^{-1} \geq N_{1} A^{-1} \geq 0
$$

which leads to the conclusion that the second splitting should be a nonnegative splitting. In the case when $N_{1} M_{1}^{-1} \geq 0$ and $M_{2}^{-1} N_{2} \geq 0$, similar considerations allow us to conclude again that the second splitting should be a nonnegative splitting.

Since for each weak nonnegative splitting we have $A^{-1} N \geq M^{-1} N$ or $N A^{-1} \geq$ $N M^{-1}$, it may occur that, for instance, $N A^{-1} \geq 0$ with $N M^{-1} \nsucceq 0$. Just such an 
example of splitting can be obtained using the examples of Climent and Perea [2] as follows.

$$
A=\left[\begin{array}{rrr}
1 & -2 & 1 \\
0 & 2 & -2 \\
-1 & 0 & 2
\end{array}\right]=M_{1}-N_{1}=M_{2}-N_{2}, \text { where } A^{-1}=\left[\begin{array}{rrr}
2 & 2 & 1 \\
1 & 3 / 2 & 1 \\
1 & 1 & 1
\end{array}\right]>0
$$

and

$$
\begin{aligned}
& M_{1}=\left[\begin{array}{rrr}
1 & -3 / 2 & 3 / 4 \\
0 & 2 & -201 / 100 \\
-1 & -1 / 2 & 3
\end{array}\right], N_{1}=\left[\begin{array}{rrr}
0 & 1 / 2 & -1 / 4 \\
0 & 0 & -1 / 100 \\
0 & -1 / 2 & 1
\end{array}\right] \text {, } \\
& M_{1}^{-1}=\frac{1}{696}\left[\begin{array}{lll}
999 & 825 & 303 \\
402 & 750 & 402 \\
400 & 400 & 400
\end{array}\right] \text {, } \\
& M_{2}=\left[\begin{array}{rrr}
1 & -3 / 2 & 3 / 4 \\
0 & 2 & -2 \\
0 & -1 / 2 & 3
\end{array}\right], \quad N_{2}=\left[\begin{array}{rrr}
0 & 1 / 2 & -1 / 4 \\
0 & 0 & 0 \\
1 & -1 / 2 & 1
\end{array}\right] \text {, } \\
& M_{2}^{-1}=\frac{1}{40}\left[\begin{array}{rrr}
40 & 33 & 12 \\
0 & 24 & 16 \\
0 & 4 & 16
\end{array}\right] \text {, } \\
& M_{1}^{-1} N_{1}=\frac{1}{696}\left[\begin{array}{rrr}
0 & 348 & 45 \\
0 & 0 & 309 \\
0 & 0 & 296
\end{array}\right], N_{1} M_{1}^{-1}=\frac{1}{696}\left[\begin{array}{rrr}
101 & 275 & 101 \\
-4 & -4 & -4 \\
199 & 25 & 199
\end{array}\right] \text {, } \\
& M_{2}^{-1} N_{2}=\frac{1}{20}\left[\begin{array}{rrr}
6 & 17 & 1 \\
8 & -4 & 8 \\
8 & -4 & 8
\end{array}\right], N_{2} M_{2}^{-1}=\frac{1}{40}\left[\begin{array}{rrr}
0 & 11 & 4 \\
0 & 0 & 0 \\
40 & 17 & 20
\end{array}\right] \text {, } \\
& A^{-1} N_{2}=\frac{1}{4}\left[\begin{array}{lll}
4 & 2 & 2 \\
4 & 0 & 3 \\
4 & 0 & 3
\end{array}\right] \geq A^{-1} N_{1}=\frac{1}{200}\left[\begin{array}{rrr}
0 & 100 & 92 \\
0 & 0 & 147 \\
0 & 0 & 148
\end{array}\right] \geq 0 \text {, } \\
& N_{2} A^{-1}=\left[\begin{array}{rrr}
1 / 4 & 1 / 2 & 1 / 4 \\
0 & 0 & 0 \\
5 / 2 & 9 / 4 & 3 / 2
\end{array}\right] \geq N_{1} A^{-1}=\left[\begin{array}{rrr}
1 / 4 & 1 / 2 & 1 / 4 \\
-1 / 100 & -1 / 100 & -1 / 100 \\
1 / 2 & 1 / 4 & 1 / 2
\end{array}\right] \nsupseteq 0 .
\end{aligned}
$$

In this example the first splitting is weak nonnegative of the first type with $N_{1} A^{-1} \geq 0$ but the second splitting is weak nonnegative of the second type with $A^{-1} N_{2} \geq 0$ and $N_{2} A^{-1} \geq 0$. Since in this case assumption (1.1) is satisfied and moreover $A^{-1} N_{2} \geq A^{-1} N_{1} \geq 0$, we can conclude that

$$
0.4253=\varrho\left(M_{1}^{-1} N_{1}\right)<\varrho\left(M_{2}^{-1} N_{2}\right)=0.6531 .
$$

The above result indicates existing a subclass of weak nonnegative splittings with stronger conditions $A^{-1} N \geq 0$ and $N A^{-1} \geq 0$ which leads to the following reformulation of weak nonnegative splittings. 
Definition 1.4. Let $M, N \in \mathbb{R}^{n \times n}$. Then the decomposition $A=M-N$ is called:

(c') a strict weak nonnegative splitting of $A$ if $M^{-1} \geq 0$ and either $M^{-1} N \geq 0$ (the first type) or $N M^{-1} \geq 0$ (the second type), where $A^{-1} N \geq 0$ and $N A^{-1} \geq 0$,

(c") a nonstrict weak nonnegative splitting of $A$ if $M^{-1} \geq 0$ and either $M^{-1} N \geq 0$ (the first type) or $N M^{-1} \geq 0$ (the second type), where either $A^{-1} N \geq 0$ and $N A^{-1} \nsucceq 0$, or $A^{-1} N \geq 0$ and $N A^{-1} \geq 0$, respectively.

A similar reformulation may be done for the case of weaker splittings.

In the case of the weaker condition $M_{1}^{-1} \geq M_{2}^{-1}$ the contrary behavior is observed. As is demonstrated on examples in [8], when both weak nonnegative splittings of a monotone matrix $A$ are the same type, with $M_{1}^{-1} \geq M_{2}^{-1}$ (or even $M_{1}^{-1}>M_{2}^{-1}$ ) it may occur that $\varrho\left(M_{1}^{-1} N_{1}\right)>\varrho\left(M_{2}^{-1} N_{2}\right)$. The conclusions for the case of weak nonnegative splittings are summarized in the following corollary.

Corollary 1.5. Let $A=M_{1}-N_{1}=M_{2}-N_{2}$ be two weak nonnegative splittings, where $A^{-1} \geq 0$, then:

(a) the assumption $N_{2} \geq N_{1}$ allows us to prove that $\varrho\left(M_{1}^{-1} N_{1}\right) \leq \varrho\left(M_{2}^{-1} N_{2}\right)$ if one of splittings is at least strict, this assumption may be not valid when both splittings are nonstrict of different types,

(b) if both splittings are of the same type, then the assumption $M_{1}^{-1} \geq M_{2}^{-1} \geq 0$ is not a sufficient condition for proving that $\varrho\left(M_{1}^{-1} N_{1}\right) \leq \varrho\left(M_{2}^{-1} N_{2}\right)$.

For nonnegative splittings we have the following result.

TheOREM 1.6. [8] Let $A=M_{1}-N_{1}=M_{2}-N_{2}$ be two nonnegative splittings of $A$, where $A^{-1} \geq 0$. If $M_{1}^{-1} \geq M_{2}^{-1}$, then

$$
\varrho\left(M_{1}^{-1} N_{1}\right) \leq \varrho\left(M_{2}^{-1} N_{2}\right) .
$$

If $A^{-1}>0$ and $M_{1}^{-1}>M_{2}^{-1}$, then

$$
\varrho\left(M_{1}^{-1} N_{1}\right)<\varrho\left(M_{2}^{-1} N_{2}\right) .
$$

But for different types of weak nonnegative splittings there is the similar result.

THEOREM 1.7. [8] Let $A=M_{1}-N_{1}=M_{2}-N_{2}$ be two weak nonnegative splittings of different types, that is, either $M_{1}^{-1} N_{1} \geq 0$ and $N_{2} M_{2}^{-1} \geq 0$ or $N_{1} M_{1}^{-1} \geq 0$ and $M_{2}^{-1} N_{2} \geq 0$, where $A^{-1} \geq 0$. If $M_{1}^{-1} \geq M_{2}^{-1}$, then

$$
\varrho\left(M_{1}^{-1} N_{1}\right) \leq \varrho\left(M_{2}^{-1} N_{2}\right) .
$$

If $A^{-1}>0$ and $M_{1}^{-1}>M_{2}^{-1}$, then

$$
\varrho\left(M_{1}^{-1} N_{1}\right)<\varrho\left(M_{2}^{-1} N_{2}\right) .
$$

Remark 1.8. Obviously, the case of two mixed splittings of $A=M_{1}-N_{1}=$ $M_{2}-N_{2}$ (i.e., when one of them is nonnegative and the second is weak nonnegative) is fulfilled by the assumptions of Theorem 1.7.

Theorem 1.7 is valid independently if the splittings are strict or nonstrict. As is well known for weak nonnegative splittings the condition $N_{2} \geq N_{1}$ implies the condition $M_{1}^{-1} \geq M_{2}^{-1} \geq 0$ but the contrary statement may be not valid [8]. In 
the example considered above we have $M_{1}^{-1}>M_{2}^{-1} \geq 0$, which implies the strict inequality in (1.2).

In the case of the Berman and Plemmons' weak regular splitting [1], corresponding to the weak nonnegative splitting of the first type, Elsner [3] showed that the assumption $M_{1}^{-1} \geq M_{2}^{-1} \geq 0$ may be not a sufficient hypothesis for ensuring the inequality $\varrho\left(M_{1}^{-1} N_{1}\right) \leq \varrho\left(M_{2}^{-1} N_{2}\right)$ and he stated result of Theorem 1.7 for the case when one of the splittings is regular. This means that Elsner restored the need of the condition $N M^{-1} \geq 0$ sticking originally in the Ortega and Rheinboldt's definition of weak regular splitting. It is evident that Elsner's result is a particular case of Theorem 1.7.

The Ortega and Rheinboldt's definition of weak regular splitting is used by Lanzkron, Rose, and Szyld [4] and they have proved the following theorem.

Theorem 1.9. ([4, Theorem 3.1]) Let $A=M_{1}-N_{1}=M_{2}-N_{2}$ be convergent weak regular splittings (that is, nonnegative splittings) such that

$$
M_{1}^{-1} \geq M_{2}^{-1},
$$

and let $x$ and $z$ be the nonnegative Frobenius eigenvectors of $M_{1}^{-1} N_{1}$ and $M_{2}^{-1} N_{2}$, respectively. If $N_{2} z \geq 0$ or if $N_{1} x \geq 0$ with $x>0$, then

$$
\varrho\left(M_{1}^{-1} N_{1}\right) \leq \varrho\left(M_{2}^{-1} N_{2}\right) \text {. }
$$

As can be deduced from Corollary 1.3, the term "convergent" is equivalent to the assumption that $A^{-1} \geq 0$. Since $M^{-1} \geq 0, M^{-1} N \geq 0$ and $\varrho\left(M^{-1} N\right)<1$ by the assumption, then

$$
A^{-1}=\left[I-M^{-1} N\right]^{-1} M^{-1}=\left[I+M^{-1} N+\left(M^{-1} N\right)^{2}+\left(M^{-1} N\right)^{3}+\ldots\right] M^{-1} \geq 0
$$

and conversely, if $A^{-1} \geq 0$, then $\varrho\left(M^{-1} N\right)<1$.

As follows from Theorem 1.6, the hypothesis $M_{1}^{-1} \geq M_{2}^{-1}$ is a sufficient condition in this theorem and the assumptions $N_{2} z \geq 0$ or $N_{1} x \geq 0$ with $x>0$ are superfluous because each one follows from the properties of nonnegative splittings. For each nonnegative splitting of $A=M-N$, where $A^{-1} \geq 0$ and $1>\lambda=\varrho\left(M^{-1} N\right) \geq 0$, one can write

$$
M^{-1} N x=\lambda x, \text { where } x \geq 0
$$

or equivalently

$$
\begin{aligned}
N x & =\lambda M x, \\
N M^{-1} M x & =\lambda M x, \\
N M^{-1} y & =\lambda y .
\end{aligned}
$$

Since $N M^{-1}$ is a nonnegative matrix, then its eigenvector $y=M x \geq 0$, hence

$$
N x=\lambda y \geq 0 .
$$


Thus, this theorem supplied with additional but completely superfluous conditions, is equivalent to Theorem 1.6.

Remark 1.10. We point out that Theorem 1.9 was restated in [5, Theorem 3.5] but for weak nonnegative splittings of the first type. In that reformulation, the conditions $N_{2} z \geq 0$ or $N_{1} x \geq 0$ are not superfluous.

Acknowledgement. Thanks are due to Professor Daniel Szyld for making accessible the example derived by Climent and Perea [2], inspiring us to a further extention of the class of splittings.

\section{REFERENCES}

[1] A. Berman and R. J. Plemmons. Nonnegative matrices in the mathematical sciences. Academic Press, New York, 1979. Reprinted by SIAM, Philadelphia, 1994.

[2] J.-J. Climent and C. Perea. Some comparison theorems for weak nonnegative splittings of bounded operators. Linear Algebra Appl., 275-276:77-106, 1998.

[3] L. Elsner Comparisons of weak regular splittings and multisplitting methods. Numer. Math., 56:283-289, 1989.

[4] P. J. Lanzkron, D. J. Rose, and D. B. Szyld. Convergence of nested classical iterative methods for linear systems. Numer. Math., 58:685-702, 1991.

[5] I. Marek and D. B. Szyld. Comparison theorems for weak splittings of bounded operators. Numer. Math., 58:387-397, 1990.

[6] J. M. Ortega and W. Rheinboldt. Monotone iterations for nonlinear equations with applications to Gauss-Seidel methods. SIAM J. Numer. Anal., 4:171-190, 1967.

[7] R. S. Varga. Matrix iterative analysis. Prentice Hall, Englewood Cliffs, N.J., 1962. Second edition, revised and expanded, Springer, Berlin, Heidelberg, New York, 2000.

[8] Z. I. Woźnicki. Nonnegative splitting theory. Japan J. Industr. Appl. Math., 11:289-342, 1994.

[9] Z. I. Woźnicki. Conditions for convergence and comparison. In Proc. 15th IMACS World Congress on Scientific Computation, Modelling and Applied Mathematics, Vol. 2, Numerical Mathematics, A.Sydov, ed., pp. 291-296. Wissenschaft and Technik Verlag, Berlin, 1997.

[10] Z. I. Woźnicki. Basic comparison theorems for weak and weaker matrix splittings. Electron. J. Linear Algebra, 8:53-59, 2001. 